\title{
Hepatectomía derecha tras extirpación de tumor de la ampolla de Vater*
}

\author{
Drs. ÁlVARO DÍAZ DE LIAÑO A. ${ }^{1}$, CONCEPCIÓN YÁRNOZ I. ${ }^{1}$, SHERBOURNE VIANA H. ${ }^{2}$, \\ CRISTINA ARTIEDA S. ${ }^{1}$, ALICIA ARTAJONA R. ${ }^{1}$, GREGORIO GONZÁLEZ A. ${ }^{1}$, \\ LORANS FERNÁNDEZ R. ${ }^{1}$, HÉCTOR ORTIZ H. ${ }^{1}$ \\ 1 Servicio de Cirugía General, Hospital Universitario Virgen del Camino, Pamplona, Navarra, España. \\ 2 Clínica Quirúrgica F, Hospital de Clínicas, Montevideo, Uruguay.
}

\begin{abstract}
Right hepatectomy after excision of a carcinoma of the ampulla of Vater. Report of one case

Occasionally hepatectomy for metastases of ampulla of Vater carcinoma can result in a better survival and quality of life of patients. We report a 67 years old male subjected to a duodenopancreatectomy for a carcinoma of the ampulla of Vater that required afterwards a right hepatectomy for metastases. Twenty one months after the second operation and 42 months after the first operation, that patient is asymptomatic and without evidences of relapse.
\end{abstract}

Key words: Ampulla of Vater, carcinoma, hepatectomy.

\section{Resumen}

El tratamiento quirúrgico del carcinoma de ampolla de Vater presenta mejores resultados oncológicos que los del resto de los tumores periampulares. En casos seleccionados, la resección hepática por metástasis de carcinoma de ampolla de Vater extirpado previamente, puede proporcionar supervivencias prolongadas y con buena calidad de vida. Presentamos un paciente de 67 años tratado con duodenopancreatectomía por cáncer de la ampolla de Vater y posteriormente con hepatectomía derecha por metástasis. A los 42 y 21 meses de la primera y segunda intervención respectivamente, permanece asintomático y sin evidencia de enfermedad.

Palabras clave: Hepatectomía, cáncer ampolla Vater, duodenopancreatectomía.

\section{Introducción}

El carcinoma de ampolla de Vater constituye el 6 al $10 \%$ del total de las neoplasias periampulares y presenta un pronóstico aceptable desde el punto de vista oncológico con resecabilidad del $80-90 \%$ y supervivencia global a 5 años del $60 \%$ en los casos extirpados ${ }^{1}$. Las dos causas más frecuentes de fallo en la evolución de los casos resecados son la recurrencia local y las metástasis.

*Recibido el 12 de Diciembre de 2008 y aceptado para publicación el 11 de Febrero de 2009.

Correspondencia: Dr. Á. Díaz de Liaño A.

C/ Pintor Maeztu n ${ }^{\circ}, 8^{\circ} \mathrm{C}$. Pamplona 31008, Navarra, España.

E-mail: adiazdea@cfnavarra.es 
En el trabajo de Hsu et al, sobre una serie de 135 pacientes con adenocarcinoma de ampolla resecados, el $23 \%$ de las recidivas se presentan a nivel hepático, seguidas de las locorregionales en un $19 \%$; la mayoría de las metástasis son múltiples o se presentan en concomitancia con recurrencia en otros sitios, lo que determina un pronóstico sombrío con supervivencias para estos pacientes menores al $1 \%$ a los 6 meses $^{2}$.

Hay pocos casos referidos en la literatura de pacientes a los que después de realizado el tratamiento quirúrgico del cáncer de ampolla de Vater se realiza rescate quirúrgico de la metástasis hepática.

Presentamos el caso clínico de un paciente, con recaída tumoral en forma de secundarismo hepático único, al que se realizó tratamiento quirúrgico con criterio oncológico y con una sobrevida libre de enfermedad prolongada.

\section{Caso clínico}

Varón de 67 años de edad intervenido quirúrgicamente en mayo de 2005 por adenocarcinoma de ampolla de Vater al que se practicó duodenopancreatectomía cefálica con preservación pilórica y linfadenectomía del hilio hepático y peripancreática. El estudio de anatomía patológica informó un adenocarcinoma de ampolla de Vater bien diferenciado de $2,5 \mathrm{~cm}$ de diámetro, con márgenes quirúrgicos libres de tumor, infiltración venosa microscópica, sin infiltración linfática ni perineural y 7 ganglios aislados de los cuales uno presentaba metástasis.

Posteriormente, se trató con seis ciclos de quimioterapia, con Gemcitabina $\left(1.000 \mathrm{mg} / \mathrm{m}^{2}\right)$, con buena tolerancia y sin complicaciones.

Se realizó seguimiento trimestral con examen clínico, determinación de marcadores tumorales, radiografía de tórax, ecografía, y tomografía computarizada abdomino-pélvica. Estando asintomático y con buen estado general, a los 18 meses se detectó un Ca19.9 de 145 U/I (0-24 U/I) con tomografía abdomino-pélvica sin evidencia de recaída de la enfermedad. A los dos meses se visualizó en la ecografía abdominal (Figura 1) y la tomografía abdominal, una imagen sólida única en el lóbulo derecho hepático, segmentos VI-VII, en íntimo contac-

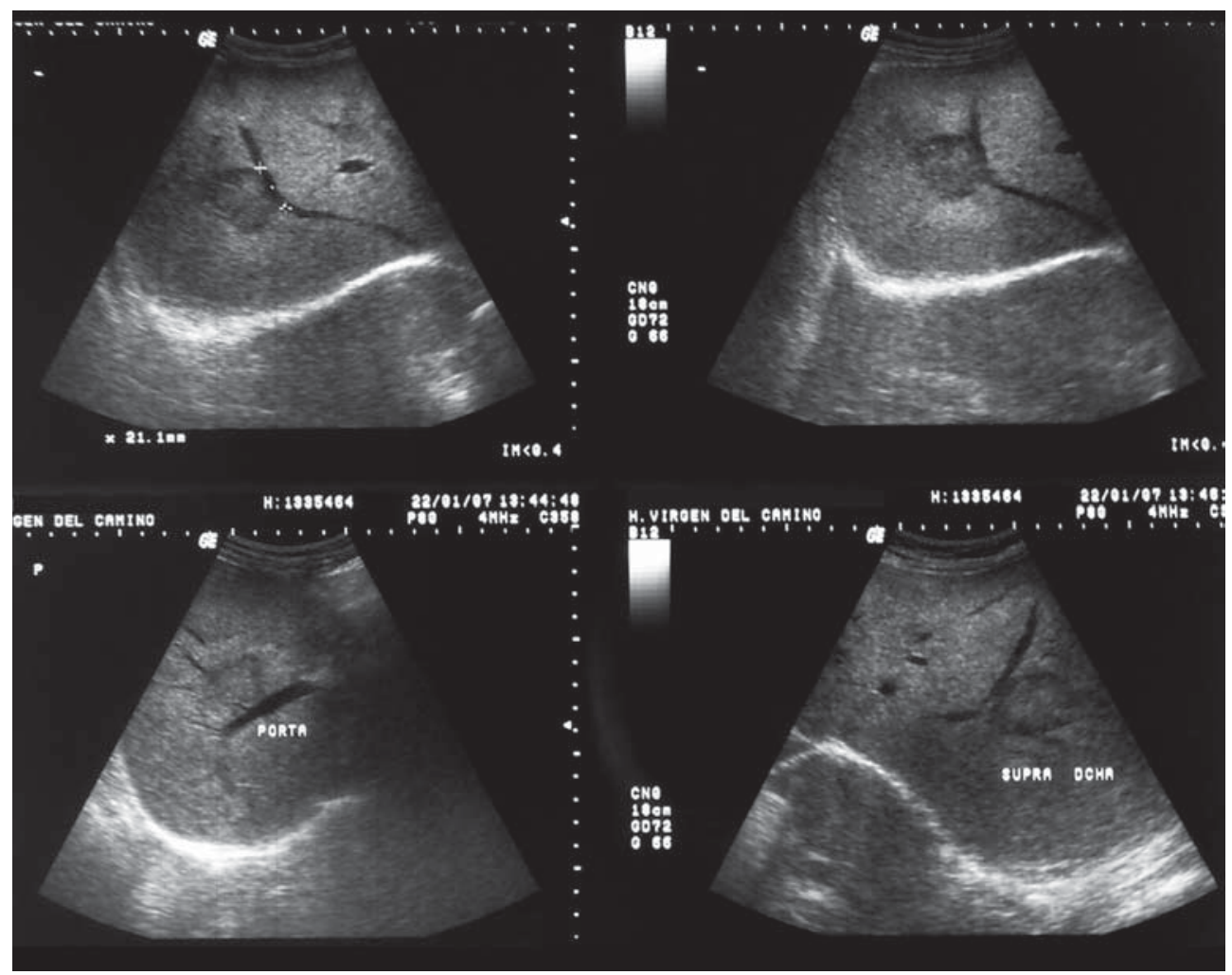

Figura 1. Ecografía abdominal que muestra la lesión a nivel hepático y la relación con estructuras vasculares. 


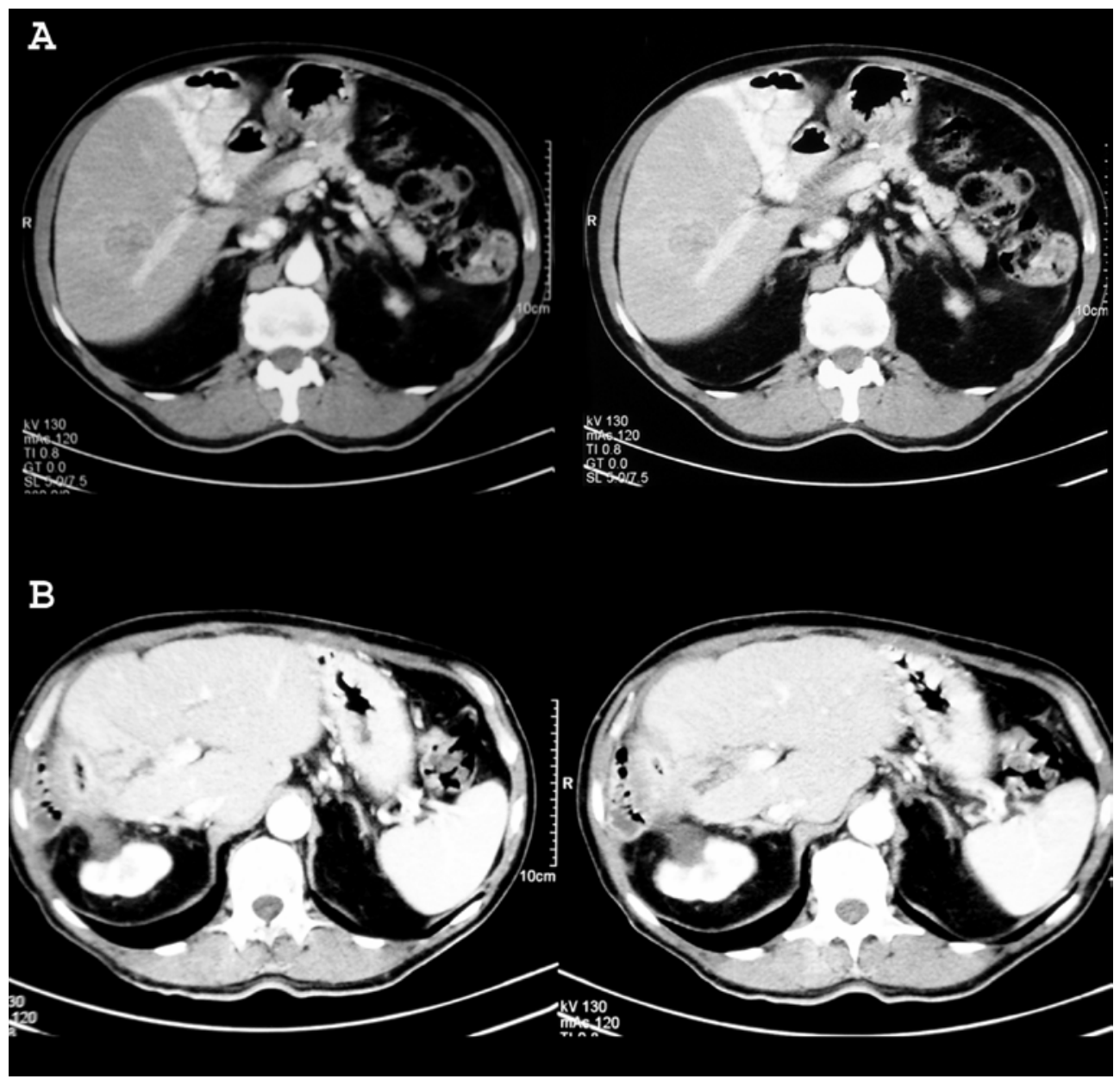

Figura 2. A) Se muestra la lesión hepática única y relación con rama derecha portal. B) Remanente hepático y ausencia de recidiva a los 21 meses.

to con rama portal y suprahepática derechas (Figura 2), y sin evidencia de adenopatías locorregionales. Dado el excelente estado general del paciente con un IMC de 24 y muy buena valoración funcional cardiovascular y respiratoria, así como las características de la recidiva y resultados oncológicos de la cirugía anterior, se decide tratamiento quirúrgico de la metástasis realizando lobectomía derecha típica en febrero de 2007. En el curso postoperatorio presentó como complicación quirúrgica un absceso subfrénico que se trató mediante antibioterapia y drenaje percutáneo. El examen histológico del hígado resecado demostró una metástasis de adenocarcinoma bien diferenciado.

A los 42 meses de la cirugía primaria y 21 meses de la cirugía de la metástasis, el paciente está asintomático y sin evidencia de recidiva de la enfermedad.

\section{Discusión}

El tratamiento quirúrgico del carcinoma de ampolla de Vater es el que proporciona los mejores resultados luego de la resección oncológica, comparándolo con el del resto de tumores periampulares, presentando un buen pronóstico tras la resección curativa con supervivencias a 5 años en diferentes series que oscilan del 40 al $60 \%{ }^{1,3}$.

Estos buenos resultados oncológicos se ven ensombrecidos cuando aparecen recidivas de la enfermedad a nivel locorregional o hepático, sitio este el más frecuente de presentación, aunque generalmente múltiple.

Estas recurrencias de la enfermedad parecen determinadas por características tumorales y factores dependientes de la cirugía inicial.

Del tumor es importante la variedad histológica 
ya que dentro de los adenocarcinomas existen dos subtipos con diferente agresividad y por lo tanto, diferente pronóstico; uno de tipo intestinal y comportamiento similar a la presentación colorrectal con mejor pronóstico, y otra variedad de peor pronóstico de tipo pancreático. También influyen otros factores como el tamaño del tumor, tomando como límite los $2 \mathrm{~cm}$, la presencia de ganglios positivos en la cirugía primaria, así como la existencia de invasión microscópica tumoral linfática y/o venosa. Cuando está presente la invasión microscópica linfática y venosa es un indicador de recidiva precoz a nivel hematógena, y cuando está presente la linfática la recurrencia será más probable a nivel ganglionar ${ }^{1,2,4,6,7}$.

En cuanto a la cirugía primaria, esta debe ser emprendida con criterio radical y curativo, siendo aceptado unánimemente en los centros especializados de alto volumen como el tratamiento oncológicamente correcto, la duodenopancreatectomía cefálica, con linfadenectomía regional que incluye los grupos del ligamento hepatoduodenal, pedículo hepático, peripancreáticos y mesentérico superior. Las linfadenectomías radicales que incluyen el tronco celíaco y latero cavo-aórticas, preconizadas por algunos cirujanos, son controvertidas y no existe consenso general ${ }^{2,4,7}$.

Del tratamiento adyuvante, los trabajos al respecto coinciden en que hacen falta estudios aleatorizados para definir qué pacientes se verán beneficiados, así como el régimen de quimioterapia más adecuado, aunque se acepta el beneficio de la quimioterapia en pacientes con alto riesgo de recaída según el estudio histológico ${ }^{2,7}$.

Por todo ello, se aconseja el seguimiento estricto de los pacientes resecados con criterio curativo con marcadores tumorales y pruebas de imagen sobre todo en los dos primeros años, ya que es en este lapso cuando aparecen en su mayor porcentaje las recidivas de la enfermedad.

La mayoría de las descripciones de cirugía de la recidiva hepática son casos aislados, aunque el buen resultado de algunos de ellos apoya la importancia del seguimiento postoperatorio de los pacientes resecados de tumores de la ampolla de Vater $^{8-10}$.

El caso presentado está asintomático y sin evidencia de enfermedad a los 42 meses de la duodenopancreatecromía cefálica y tras 21 meses de lobectomía hepática derecha por metástasis única.

Como conclusión, creemos que en casos selec- cionados, la cirugía hepática de la recidiva tras duodenopancreatectomía por cáncer de la ampolla de Vater, puede ser una alternativa terapéutica válida, aunque los buenos resultados comunicados corresponden a casos aislados.

\section{Referencias}

1. Todoroki T, Koike N, Morishita Y. Kawamoto T, Ohkohchi N, Shoda J, et al. Patterns and predictors of failure after curative resection of carcinoma of ampulla of Vater. Ann Surg Oncol 2003; 10: 1176-1183.

2. Hsu HP, Yang TM, Hsieh YH, Shan YS, Lin PW. Predictors for patterns of failure after pancreaticoduodenectomy in ampullary cancer. Ann Surg Oncol 2007; 14: 50-60.

3. Talamini M, Moesinger R, Pitt H, Sohn T, Hruban R, Lillemoe $\mathrm{K}$, et al. Adenocarcinoma of the ampulla of Vater: a 28-year experience. Ann Surg. 1997; 225: 590595.

4. Park JS, Yoon DS, Kim KS, Choi JS, Lee WJ, Chi HS, et al. Factors influencing recurrence after curative resection for ampulla of Vater carcinoma. J Surg Oncol 2007; 95: 286-290.

5. Howe J, Klimistra D, Moccia R, Conlon K, Brennan M. Factors predictive of survival in ampullary carcinoma. Ann Surg 1998; 228: 87-94.

6. Pérez-Cabrera B, Sánchez B, Luque F, Gómez E, Bravo $\mathrm{F}$, Pérez $\mathrm{F}$, y col. Tratamiento quirúrgico de los adenocarcinomas de la ampolla de Vater. Cir Esp 2000; 67: 21-24.

7. Conlon KC. Carcinoma of the ampulla of Vater: a distinct disease entity. Ann Surg Oncol 2003; 10: 1136-1137.

8. Yoshida T, Matsumoto T, Sasaki A, Bandoh T, Kawano K, Kitano S, et al. Hepatectomy for liver metastasis from ampullary cancer after pancreatoduodenectomy. Hepatogastroenterology 2002; 49: $247-$ 248.

9. Kitami E, Kurosaki I, Koyama Y, Makino H, Hatakeyama K. Long-term survival after hepatectomy for hepatic recurrence of carcinoma of the papilla of Vater. J Hepatobiliary Pancreat Surg 2005; 12: 321323.

10. Moriya T, Kimura W, Hirai I, Mizutani M, Yamamoto $\mathrm{T}$, Toya $\mathrm{R}$, et al. Twelve years survival with repeated hepatectomy and lung resection for metastasis from carcinoma of the papilla of Vater after pancreaticoduodenectomy. Hepatogastroenterology 2007; 54: 1652-1654. 